\title{
MR Myelography for the Detection of CSF-Venous Fistulas
}

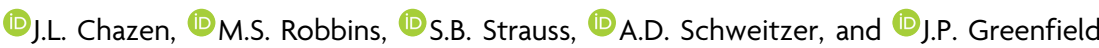

\section{ABSTRACT}

SUMMARY: CSF-venous fistula is an important treatable cause of spontaneous intracranial hypotension that is often difficult to detect using traditional imaging techniques. Herein, we describe the technical aspects and diagnostic performance of MR myelography when used for identifying CSF-venous fistulas. We report 3 cases in which the CSF-venous fistula was occult on CT myelography but readily detected using MR myelography.

ABBREVIATIONS: $\mathrm{CVF}=\mathrm{CSF}$-venous fistula; $\mathrm{SIH}=$ spontaneous intracranial hypotension; VIBE $=$ volumetric interpolated brain examination

C SF-venous fistula (CVF) is an important cause of spontaneous intracranial hypotension (SIH) and a critical diagnosis because treatment can result in a clinical cure. ${ }^{1-5}$ The fistulous connection is typically associated with a nerve root sleeve diverticulum; therefore, a detectable epidural fluid collection is often absent. Some authors advocate decubitus CT myelography to improve CVF detection, ${ }^{1,2,6}$ while others suggest that digital subtraction myelography may increase rates of fistula identification. ${ }^{7,8}$ We report surgically confirmed cases of CVF that were identified using MR myelography following the intrathecal administration of gadolinium.

\section{MR Myelography Technique}

Intrathecal gadolinium was instilled in the subarachnoid space via CT-guided lumbar puncture using a Gertie Marx Whitacrestyle spinal needle (IMD). The opening pressure was measured and recorded, and $0.5 \mathrm{~mL}$ of gadobutrol (Gadavist; Bayer Schering Pharma) mixed with $4.5 \mathrm{~mL}$ of iohexol (Omnipaque 180; GE Healthcare) was intrathecally administered. After a 1$\mathrm{mL}$ test injection, CT imaging confirmed intrathecal contrast, and $5 \mathrm{~mL}$ was instilled before the needle was removed with

Received December 25, 2019; accepted after revision February 29, 2020.

From the Departments of Radiology (J.L.C., S.B.S., A.D.S.), Neurology (M.S.R.), and Neurological Surgery (J.P.G.), Weill Cornell Medicine, NewYork-Presbyterian Hospital, New York, New York.

Please address correspondence to J. Levi Chazen, MD, Weill Cornell Medicine, NewYork-Presbyterian Hospital, Department of Radiology, 520 E. 70th St. Starr 631 New York, NY 10065; e-mail: jlc2008@med.cornell.edu; @LeviChazen

Indicates article with supplemental on-line table.

Indicates article with supplemental on-line photos.

http://dx.doi.org/10.3174/ajnr.A6521 the stylet in place. The patient was positioned in a decubitus Trendelenberg position and rolled multiple times to disperse contrast throughout the subarachnoid space. The patient was then transferred to the MR imaging suite where MR myelography was performed on a 3T Biograph mMR scanner (Siemens) using multiplanar fat-suppressed T1 sequences (On-line Table). Imaging was reviewed in real time, and delayed thin-section axial sequences were acquired as needed. MR imaging was completed approximately 1 hour after intrathecal gadolinium administration.

\section{Cases}

Case 1. A 26-year-old woman with a 3-year history of orthostatic headaches after a spontaneous onset had brain MR imaging findings characteristic of SIH, including sagging, pituitary enlargement, venous distention, small subdural collections, and pachymeningeal enhancement. A CT myelogram was unrevealing for CSF leak, and 3 epidural blood patches were unsuccessful. She subsequently developed upper extremity pain and paresthesias, leading to the discovery of a large cervicothoracic syrinx on cervical spine MR imaging (Fig 1). She was treated with a posterior fossa decompression to improve craniocervical CSF flow but obtained neither symptom nor imaging improvement. Eventually, she underwent MR myelography with an opening pressure of $<4 \mathrm{~cm} \mathrm{H}_{2} \mathrm{O}$ and was discovered to have a prominent left T7-T8 spinal meningeal diverticulum fistulizing to a left paraspinous vein draining into the azygous system. She also had early contrast opacification of her renal collecting system on the MR myelography completed approximately 1 hour after intrathecal contrast injection (Fig 2). The patient was offered and accepted surgical CVF ligation at T7-T8. The diverticulum was identified with dilated draining veins, and the entire nerve root complex with the CVF was interrupted with 


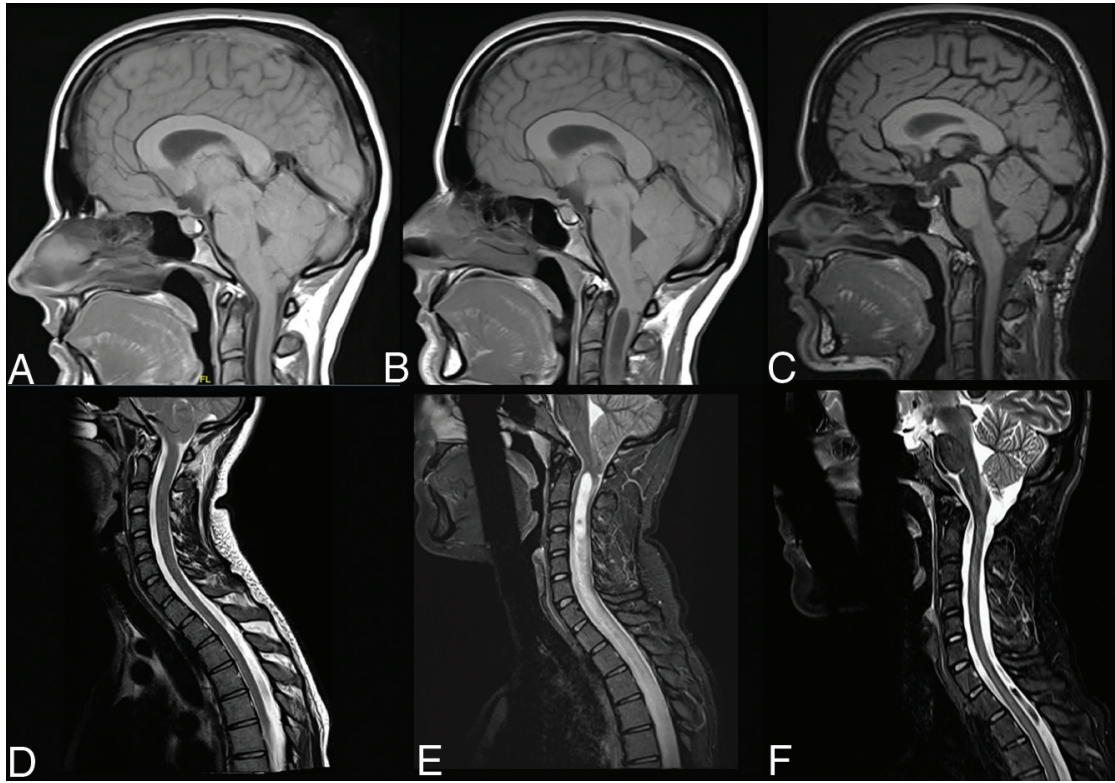

FIG 1. Sagittal T1 brain $(A-C)$ and sagittal T2-weighted cervical spine $(D-F)$ imaging. Initial MR imaging from June 2016 ( $A$ and $D$ ) shows classic intracranial findings of SIH with low-lying cerebellar tonsils, effacement of the prepontine cistern, enlarged pituitary, and brain stem sagging. Spine imaging findings are normal. Follow-up MRIs from June 2018 ( $B$ and $E$ ) show similar brain findings with interval development of a large cervicothoracic syrinx. Postoperative MRIs from June 2019 $(C$ and $F$ ) reveal complete resolution of intracranial $\mathrm{SIH}$ sequelae, with a normal position of the cerebellar tonsils and resolution of spinal syrinx.

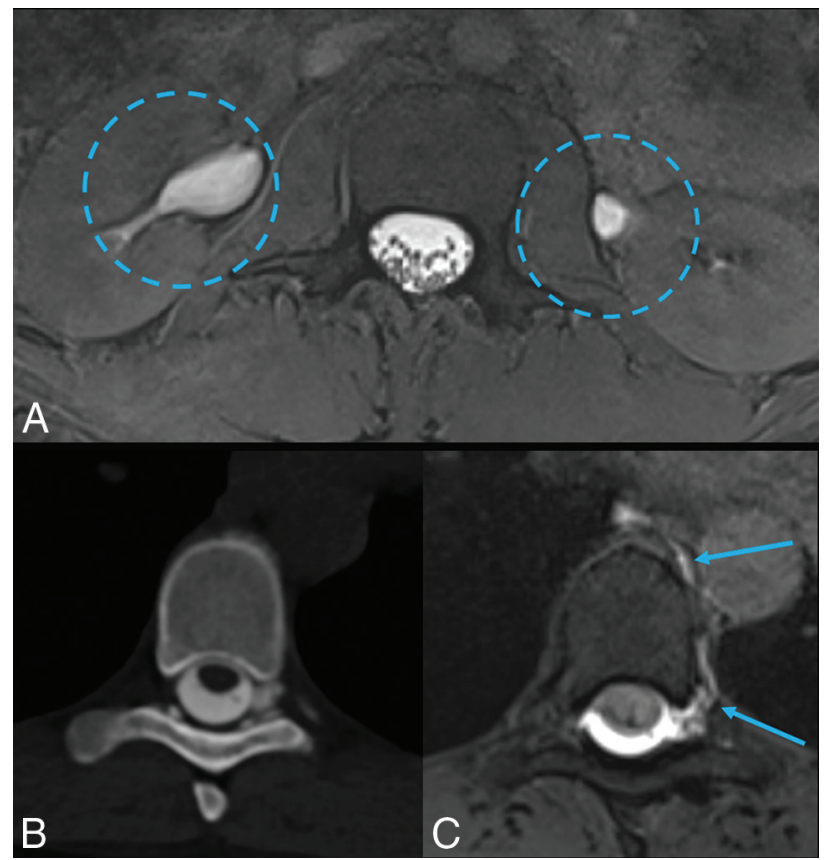

FIG 2. MR myelogram axial TI VIBE $(A)$ demonstrates early contrast opacification of the renal collecting system (dashed circles). CT myelogram (B) demonstrates an irregular left T7-T8 perineural cyst without evidence of an opacifying paraspinal vein. No renal contrast excretion was identified on CT myelography. MR myelogram axial T1 VIBE (C) shows a spinal meningeal diverticulum with an opacifying paraspinal vein (arrows) draining into the azygous system, consistent with a CSF-venous fistula. an aneurysm clip and silk suture (Online Fig 1). The patient experienced an immediate improvement in symptoms followed by a transient new headache and sixth-nerve palsy 3 days following the operation. This was presumed to be secondary to transient rebound intracranial hypertension, and her symptoms slowly resolved. Eight months postoperatively, she is without headaches, and follow-up MR imaging revealed both a dramatic resolution of brain sagging and near-complete resolution of the spinal cord syrinx (Fig 1).

Case 2. A 64-year-old man presented with a 9-month history of orthostatic headache, distorted hearing, and tinnitus with onset during Valsalva exacerbated by severe constipation. Brain imaging demonstrated a venous distention sign without other intracranial sequelae of SIH. Standard CT myelography could not identify a CSF leak, and multiple epidural blood patches were unsuccessful. An MR myelogram with an opening pressure of $9 \mathrm{~cm} \mathrm{H}_{2} \mathrm{O}$ revealed a left $\mathrm{T} 8-\mathrm{T} 9$ spinal meningeal diverticulum and adjacent opacification of a paraspinous vein (Fig 3). Small-volume contrast was also seen in the renal collecting systems. The patient was offered and accepted surgical CVF ligation at T8-T9. The diverticulum was identified with dilated draining veins, and the entire nerve root complex with the CVF was interrupted with an aneurysm clip and silk suture. The patient experienced marked symptomatic improvement with resolution of headache and tinnitus, sustained at the most recent 4-month follow-up visit.

Case 3. A 53-year-old woman presented with a 2-year history of spontaneous-onset orthostatic headache and neck pressure. Brain imaging findings were suspicious for $\mathrm{SIH}$ with sagging, pituitary enlargement, venous distention, small subdural collections, and pachymeningeal enhancement. A CT myelogram revealed multiple spinal meningeal diverticula and Tarlov cysts without evidence of spinal fluid leak, and the patient received multiple epidural blood patches with only transient improvement of symptoms. An MR myelogram with an opening pressure of $<4 \mathrm{~cm} \mathrm{H}_{2} \mathrm{O}$ revealed a left T9-T10 spinal meningeal diverticulum and an opacified left paraspinous vein (On-line Fig 2). The patient was offered and accepted surgical CVF ligation at T9T10. The diverticulum was identified with dilated draining veins, and the entire nerve root complex with the CVF was interrupted with an aneurysm clip and silk suture. The patient reported an immediate improvement in symptoms with return to baseline and resolution of headache, sustained for 7 months at the most recent follow-up visit.

AJNR Am J Neuroradiol 41:938-40 May 2020 www.ajnr.org 


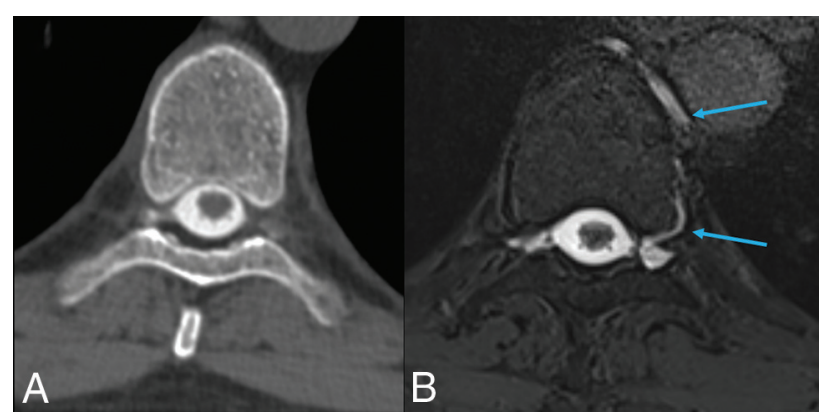

FIG 3. $C T$ and $M R$ myelogram images from case 2 . $A, C T$ myelogram reveals perineural cysts at T8-T9 without opacification of the leftsided cyst. $B$, MR myelogram axial T1 VIBE demonstrates opacification of the left-sided spinal meningeal diverticulum and left paraspinal vein draining into the azygous system (arrows), consistent with a CSFvenous fistula.

\section{DISCUSSION}

CSF-venous fistulas are an under-recognized cause of SIH. Improved detection using advanced imaging techniques may increase our sensitivity in detecting CSF leaks. CT myelography may detect CVF, and the technique can be augmented with decubitus positioning and provocative maneuvers to increase CSF pressure. ${ }^{5,9}$ Digital subtraction myelography has also been used to identify CSF-venous fistulas. However, both of these techniques require substantial ionizing radiation exposure to the patient, and the digital subtraction myelography technique is operator-dependent with different techniques described in the literature. ${ }^{7,8}$ Intrathecal administration of gadolinium is an off-label use in the United States but is well-tolerated and frequently used for clinical and research applications. ${ }^{10,11}$ However, while no long-term harmful effects from intrathecal gadolinium are known, this has not been extensively studied. Only a macrocyclic gadolinium agent (gadobutrol) was used in this study, which has a weaker association with deposition compared with linear gadolinium agents. ${ }^{12}$ MR myelography eliminates the ionizing radiation exposure aside from any used for lumbar puncture. Additionally, multiple imaging sequences can be repeated as necessary to confirm a suspected fistula. Furthermore, while the attenuation of intrathecal CT contrast fades rapidly within an hour of intrathecal administration, intrathecal gadolinium maintains robust T1-shortening MR imaging signal for hours after administration. We found the axial T1 fat-suppressed volumetric interpolated brain examination (VIBE) imaging sequences to be particularly helpful. These may help highlight a fistula on MR myelography that may be less apparent on other imaging modalities. The technique is further bolstered by the improved soft-tissue contrast of MR imaging and the prominent signal intensity of gadolinium on fat suppressed T1-weighted images. MR myelography has been shown previously to detect leaking gadolinium from perineural cysts. $^{13}$

Outstanding questions remain regarding the diagnostic performance of MR myelography and other myelographic techniques in the detection of CVF because no known criterion standard is available. We report 3 patients who had unrevealing CT myelograms in whom CVF was detected on MR myelography, all of whom experienced marked clinical improvement following ligation of the suspected fistula. This report raises important questions for further study regarding the optimal technique for imaging patients with suspected CSF leak.

In conclusion, MR myelography with intrathecal gadolinium may identify CSF-venous fistulas in patients without readily apparent fistulas on CT myelography. Further study is needed to confirm these promising findings.

Disclosures: Dr. Chazen—RELATED: Grant: Insightec, Inc., Alzheimer's Drug Discovery Foundation.

\section{REFERENCES}

1. Kranz PG, Gray L, Amrhein TJ. Decubitus CT myelography for detecting subtle CSF leaks in spontaneous intracranial hypotension. AJNR Am J Neuroradiol 2019;40:754-56 CrossRef Medline

2. Kranz PG, Amrhein TJ, Gray L. CSF venous fistulas in spontaneous intracranial hypotension: imaging characteristics on dynamic and CT myelography. AJR Am J Roentgenol 2017;209:1360-66 CrossRef Medline

3. Kumar N, Diehn FE, Carr CM, et al. Spinal CSF venous fistula: a treatable etiology for CSF leaks in craniospinal hypovolemia. Neurology 2016;86:2310-12 CrossRef Medline

4. Schievink WI, Moser FG, Maya MM. CSF-venous fistula in spontaneous intracranial hypotension. Neurology 2014;83:472-73 CrossRef Medline

5. Duvall JR, Robertson CE, Cutsforth-Gregory JK, et al. Headache due to spontaneous spinal cerebrospinal fluid leak secondary to cerebrospinal fluid-venous fistula: case series. Cephalalgia 2019;39:1847-54 CrossRef Medline

6. Kranz PG, Amrhein TJ, Schievink WI, et al. The "hyperdense paraspinal vein" sign: a marker of CSF-venous fistula. AJNR Am J Neuroradiol 2016;37:1379-81 CrossRef Medline

7. Schievink WI, Maya MM, Moser FG, et al. Lateral decubitus digital subtraction myelography to identify spinal CSF-venous fistulas in spontaneous intracranial hypotension. J Neurosurg Spine 2019 Sep 1-4. [Epub ahead of print] CrossRef Medline

8. Farb RI, Nicholson PJ, Peng PW, et al. Spontaneous intracranial hypotension: a systematic imaging approach for CSF leak localization and management based on MRI and digital subtraction myelography. AJNR Am J Neuroradiol 2019;40:745-53 CrossRef Medline

9. Griffin AS, Lu L, Peacock S, et al. CSF volume provocation maneuvers during lumbar puncture as a possible predictive tool for diagnosing spontaneous intracranial hypotension. Clin Neurol Neurosurg 2019;186:105552 CrossRef Medline

10. Dillon WP. Intrathecal gadolinium: its time has come? AJNR Am J Neuroradiol 2008;29:3-4 CrossRef Medline

11. Akbar JJ, Luetmer PH, Schwartz KM, et al. The role of MR myelography with intrathecal gadolinium in localization of spinal CSF leaks in patients with spontaneous intracranial hypotension. AJNR Am J Neuroradiol 2012;33:535-40 CrossRef Medline

12. Rowe SK, Rodriguez D, Cohen E, et al. Switching from linear to macrocyclic gadolinium-based contrast agents halts the relative T1weighted signal increase in deep gray matter of children with brain tumors: a retrospective study. J Magn Reson Imaging 2020;51:288-95 CrossRef Medline

13. Chazen JL, Talbott JF, Lantos JE, et al. MR myelography for identification of spinal CSF leak in spontaneous intracranial hypotension. AJNR Am J Neuroradiol 2014;35:2007-12 CrossRef Medline 